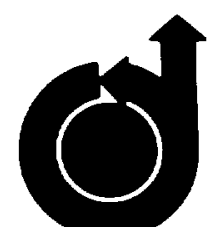

AIAA 81-0337R

Flight, Wind Tunnel, and Numerical

Experiments with a Slender Cone

at Incidence

D. J. Peake, D. F. Fisher, D. S. McRae 


\title{
Flight, Wind Tunnel, and Numerical Experiments with a Slender Cone at Incidence
}

\author{
David J. Peake* \\ NASA Ames Research Center, Moffett Field, California \\ David F. Fishert \\ NASA Dryden Flight Research Center, Edwards, California \\ and \\ David S. McRaeł \\ North Carolina State University, Raleigh, North Carolina
}

\begin{abstract}
The three-dimensional leeward separation about a 5-deg semiangle cone at 11 deg angle of incidence was investigated in night, in the wind tunnel, and by numerical computations. The test conditions were Mach numbers of $0.6,1.5$, and 1.8 at Reynolds numbers between 7 and 10 million based on freestream conditions and a $76.2-\mathrm{cm}(30-\mathrm{in}$.) length of surface. The surface pressure conditions measured included those of fluctuating and mean static, as well as recovery pressures generated by obstacle blocks to provide skin friction and separationline locations. The mean static pressures from night and wind tunnel were in reasonably good agreement. The computed results gave the same distributions, but were slightly more positive in magnitude. The experimentally measured primary and secondary separation line locations compared closely with computed results. There were substantial differences in level between the surface root-mean-square pressure nuctuations obtained in flight and in the wind tunnel, due, it is thought, to a relatively high acoustic disturbance level in the tunnel compared with the quiescent atmospheric conditions in flight.
\end{abstract}

\section{Nomenclature}

\begin{tabular}{|c|c|}
\hline $\begin{array}{l}\text { CF } \\
\text { COMP }\end{array}$ & $\begin{array}{l}=\text { skin friction coefficient }=\tau_{w} / 0.7 p_{\infty} M_{\infty}^{2} \\
=\text { computed }\end{array}$ \\
\hline $\mathrm{CP}$ & $\begin{aligned}= & \text { local static pressure coefficient at cone sur- } \\
& \text { face }=\left(p_{w}-p_{\infty}\right) / 0.7 p_{\infty} M_{\infty}^{2}\end{aligned}$ \\
\hline $\mathrm{CPB}$ & $\begin{aligned}= & \text { obstacle block pressure coefficient at cone } \\
& \text { surface }=\left(p_{B}-p_{\infty}\right) / 0.7 p_{\infty} M_{\infty}^{2}\end{aligned}$ \\
\hline CPP & $\begin{aligned}= & \text { computed pitot pressure coefficient }=\left(p_{p}-\right. \\
& \left.p_{\infty}\right) / 0.7 p_{\infty} M_{\infty}^{2}\end{aligned}$ \\
\hline CPRMS & $\begin{array}{l}=\text { root-mean-square pressure fluctuation at cone } \\
\text { surface relative to freestream dynamic } \\
\text { pressure }=\left(\langle p\rangle / q_{\infty}\right)\end{array}$ \\
\hline FLT & $=$ flight experiment \\
\hline $\boldsymbol{H}$ & $\begin{array}{l}=\text { height above cone surface divided by local radius } \\
\text { of cone }\end{array}$ \\
\hline$M_{\infty}$ & $=$ freestream Mach number \\
\hline$p_{\text {TOT }_{\infty}}$ & $\begin{array}{l}=\text { freestream stagnation pressure } \\
=\text { Reynolds number based on freestream con- } \\
\text { ditions and distance } x \text { from cone vertex }\end{array}$ \\
\hline$R N$ & $\begin{array}{l}=\text { unit Reynolds number, based on freestream } \\
\text { conditions }\end{array}$ \\
\hline 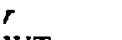 & $=$ distance along ray from cone apex \\
\hline WT & $=$ wind tunnel experiment \\
\hline$x$ & $=$ distance from apex along axis of cone \\
\hline$\alpha$ & $=$ angle of incidence \\
\hline$\beta$ & $=$ angle of sideslip \\
\hline $\begin{array}{l}\theta \\
\theta\end{array}$ & $=$ angle subtended at cone apex by conical ray \\
\hline $\begin{array}{l}\theta_{c} \\
\mu_{t}\end{array}$ & $\begin{array}{l}=\text { semiapex angle of cone } \\
=\text { eddy } y i s c o s i t y\end{array}$ \\
\hline$\rho$ & $=$ density \\
\hline & $=$ resultant shear stress ma \\
\hline
\end{tabular}

Presented as Paper 81-0337 at the AIAA 19th Aerospace Sciences Meeting, St. Louis, Mo., Jan. 12-15, 1981; submitted Feb. 23, 1981; revision received Feb. 1, 1982. This paper is declared a work of the U.S. Government and therefore is in the public domain.

- Research Assistant to Center Director. Member AIAA.

†Aerospace Engineer. Member AlAA.

$\ddagger$ Associate Professor. Member AIAA. $\phi$ Subscripts

$B \quad=$ obstacle block

$S_{1} \quad=$ primary separation line

$S_{2} \quad=$ secondary separation line

$l, 2,3=r, \theta, \phi$ directions, respectively, of spherical coordinate system

$\infty \quad=$ freestream

$w \quad$ wall

\section{Introduction}

Conical Flowfields

PHE objective of the present series of interactive experiments in flight, in the wind tunnel, and by numerical simulation, is to use the essentially conical three-dimensional separated flowfield about a sharp circular cone to provide a fundamental understanding of the mechanism of threedimensional separated flow in general and to yield criteria of performance for a common forebody shape used in the design of supersonic flight vehicles.

Next to the flow about a lifting slender delta wing, the flow about a slender cone at incidence is the simplest threedimensional body flowfield that may be envisaged. When viscosity is neglected, the flowfield about the cone in a supersonic freestream has the unique property that no changes in flow quantities occur along rays emanating from the cone apex. The stream surfaces projected on to concentric spheres centered at the apex (called conical flow streamlines) are then similar. In practice, experimenters ${ }^{1-3}$ have demonstrated that many features of the viscous/inviscid interacting flowfield, such as bow shock-wave locations, circumferential pressure distributions, normal force coefficients, circumferential positions of separation lines, and surface shear stress directions, are conical or nearly so. The growth of a laminar viscous layer cannot be conical, because it develops according to $r^{0.5}$. In a fully turbulent now, the exponent of $r$ is nearer unity, implying a flowfield very close to conical 
conditions. If the Reynolds number is sufficiently high so that transition occurs in proximity to the apex, the near-conical nature of the experimentally measured flow ${ }^{1-3}$ demonstrates a virtual absence of length effects in the streamwise direction: the flow is dominated completely by the circumferential pressure field. Thus the characteristics of these flowfields can be determined through measurement or by computation at essentially one streamwise station. In fully turbulent ${ }^{1-3}$ and fully laminar ${ }^{4}$ subsonic freestream flow, even though base and thickness effects become measurable, the circumferential pressure gradients still dominate to the extent that virtual conicity of the separation lines and shear stress directions is still maintained.

\section{Symmetric Three-Dimensional Separated Flows on the Leeward} of Cones

About the cone, the three-dimensional separation zone may be precipitated at circumferential angles, $\phi$, of $120 \mathrm{deg}$ or greater (measured from the windward ray), depending on relative incidence (angle of incidence, $\alpha$, divided by cone seminose angle, $\theta_{c}$ ), Mach number, and Reynolds number (see Fig. 63 in Ref. 5). Provided that the viscous flow is either all laminar or completely turbulent from the apex, the windward boundary layers separate from (primary) separation lines that are along conical rays, rolling up into wellorganized vortex structures situated close to the leeward meridian. The new leeward boundary layers grow outboard on each side of the leeward meridian by the induced swirling motion of the primary vortices. At sufficiently high angles of incidence, they also separate from additional conical separation lines to form small secondary vortices that are tucked beneath and are of opposite rotation to the primary vortices. Whether, for a sharp apex, the separation lines (which are particular skin-friction lines) emanate from either the nodal point of attachment at the apex, or from saddle points very close to the nose in the continuous pattern of skinfriction lines, has not been resolved..$^{5-7}$ Sequences of plausible skin-friction line patterns with saddle singular points in the vicinity of the nose as angle of incidence is increased are drawn in Ref. 5 .

The structure of the symmetric separated flow about a slender cone at incidence with both primary and secondary vortices on the leeward has been well established in experiments ${ }^{1 \cdot 3}$ and computations ${ }^{8}$ and is illustrated in Fig. 1. This drawing shows a conical flow projection with the zero velocity points (that is, singular points in this projection) that govern the pattern of the conical-flow streamlines. The shear layers departing from the surface at the three-dimensional separation lines are depicted by their dividing surfaces (median lines) starting at half-saddle points, $S^{\prime}$, and coiling into vortices, the nodal foci $N$. The remaining half-saddle points $S^{\prime}$ denote attachments to the surface in this projection. Above the primary vortices in the plane of the leeward meridian is an enclosing saddle point $S$ and a node $N$. This node has been called the "vortical singularity," being the point in the conical flow projection where the majority of conical streamlines in the external flow "disappear." In the three-dimensional flow, of course, all flow at such singular points is concentrated along a conical ray.

Figure 1 presents the conceptual model of the flowfield that has been investigated. The experiments ${ }^{9}$ have proceeded under subsonic and supersonic freestream conditions $\left(M_{\infty}=0.6,1.5\right.$, and 1.8$)$ and an angle of incidence of $11 \mathrm{deg}$ (relative incidence of 2.2 ). The same sharp 5-deg semiangle cone and instrumentation were used both in flight and in the wind tunnel. An important objective of both the flight and wind tunnel experiments was to determine the effects of the different perturbation levels in each regime on the development of the separated flow zones. Numerical computations were run for the Mach number 1.5 and 1.8 conditions with a code utilizing the conically symmetric NavierStokes equations. ${ }^{10}$ Details of the cone surface conditions beneath the turbulent viscous flow are presented herein at respective Reynolds numbers of typically 7 and 10 million for the wind tunnel and flight measurements, and are compared with computations. These Reynolds numbers are based on freestream conditions and a $76.2-\mathrm{cm}(30$-in.) axial length to the first measuring station on the cone surface.

\section{Experimental Methods}

\section{Slender Cone Model}

To reduce to a minimum the chance of spurious results, the same 5-deg semiangle sharp cone with the same instrumentation installed within the cone was tested sting mounted from an F-15 airplane (Fig. 2) and in the Ames $6 \times 6$ -

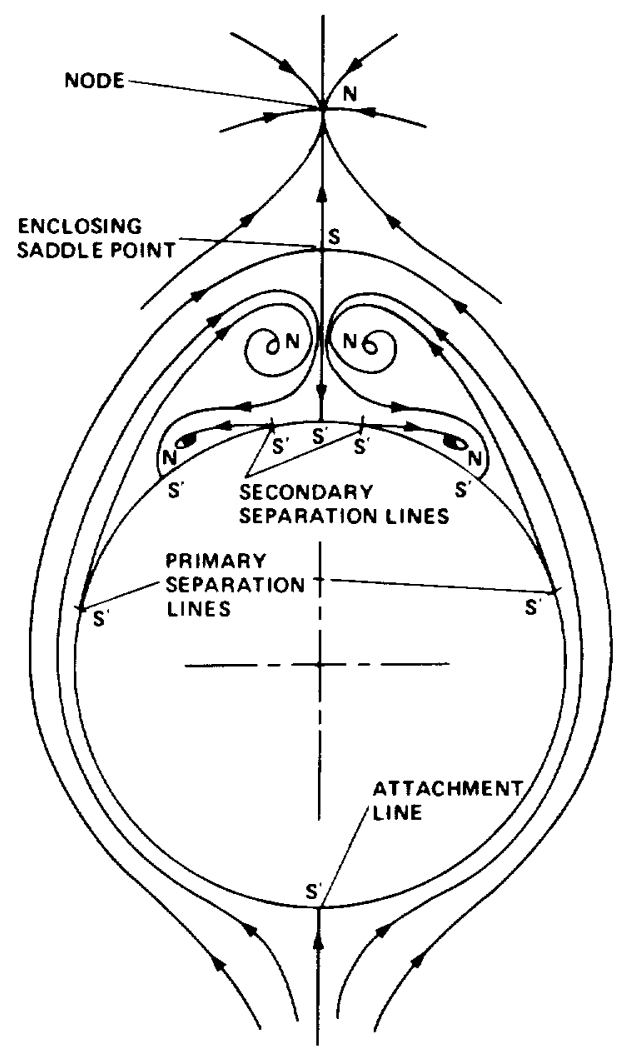

Fig. 2 Five-deg semiapex angle cone sting-mounted from nose of $F$ 15 airplane.

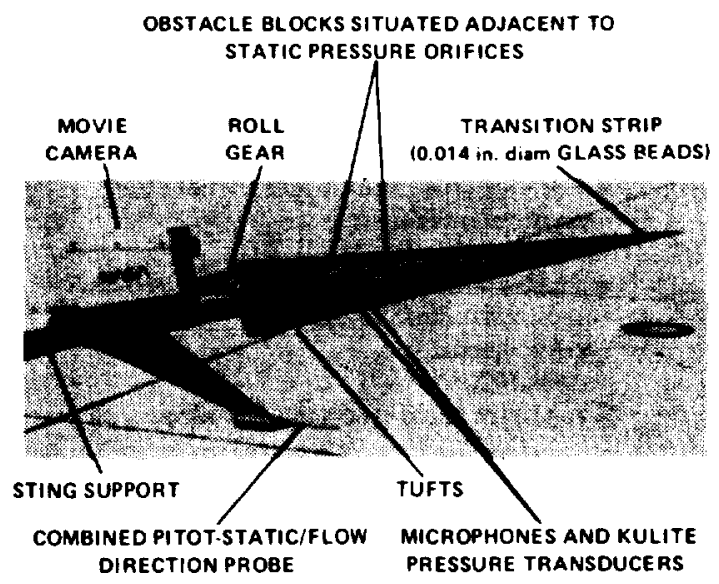

Fig. 1 Flat projection of three-dimensional stream surfaces intersecting sphere centered on cone vertex with both primary and secondary separations. 
ft $(1.8 \times 1.8-\mathrm{m})$ Wind Tunnel. The overall length of the cone was $113 \mathrm{~cm}$ (44.5 in.); it was fabricated from stainless steel with a surface smoothness better than $0.4 \mu \mathrm{m}(15 \mu \mathrm{in}$.$) and a$ tip radius of approximately $0.1 \mathrm{~mm}(0.004 \mathrm{in}$.). The cone surface was conical within $\pm 0.01 \mathrm{deg}$. A transition strip composed of nominal $0.4-\mathrm{mm}(0.014-\mathrm{in}$.) diameter glass beads was affixed to the cone at a station $12.5 \mathrm{~cm}(4.9 \mathrm{in}$.) from the apex, since some earlier and preliminary flight measurements at angle of incidence had indicated that effects of natural transition could be measured as far back as $46 \mathrm{~cm}$ (18 in.) from the tip. A description of the cone static pressure and microphone instrumentation is provided in Ref. 9.

A roll gear was mounted at the base of the cone to rotate the cone and hence the instrumentation \pm 180 deg beneath the separated flow. When the separation lines are symmetrically disposed with respect to the leeward meridian at moderate angles of incidence, it has been proved that the flowfield is insensitive to the angle of roll of the body.111 Only at elevated angles of incidence, typically greater than a relative incidence of 2.5 , is the leeward flowfield development asymmetric. ${ }^{3}$ Imperfections at the nose then cause changes in the flow direction as the model is rotated about its axis. Hence, since the cone incidence is much lower than the angle at which the onset of asymmetry occurs, the procedure of rolling the cone through the given range of $\phi$ is a viable experimental technique. This facilitates the mapping of the pressure distribution around the entire circumference by using just one static pressure orifice and one microphone or Kulite pressure transducer. The utilization of several measuring locations, both static and microphone, in a particular cross section of the body, permits a large degree of redundancy in the results.

\section{Flight Technique}

During flight, data from the combined pitot-static/flow direction probe (see Fig. 2)-aircraft-indicated Mach number and differential pressures for cone incidence and cone sideslip-were "downlinked" (transmitted) to a groundbased computer, processed in real time, then "uplinked" back to the aircraft. Special high resolution displays utilizing the uplinked information for Mach number, cone angle of incidence, and cone angle of sideslip were used in which the pilot centered the display needle to achieve the desired flight conditions. Display sensitivities and offsets of these special aircraft instruments were handled by the ground-based computer and test engineer. The test engineer also rotated the cone from the ground station to obtain the circumferential static and microphone pressure distributions.

The cone angle of incidence with respect to the aircraft axis was fixed on the ground and was not adjustable in flight. To account for any change in aircraft trim angle of incidence resulting from fuel depletion, the pilot flew the aircraft in a banked turn, holding Mach number, cone incidence, and cone angle of sideslip at the desired conditions.

\section{Turbulence Model Used in the Numerical Computations}

Details of the numerical computation technique and the auxiliary conditions are explained in Ref. 10, where the conical flow approximations to the full set of Navier-Stokes equations are written. The eddy viscosity model used for closure is reiterated here, however, as its circumferential distribution must be tailored on the leeward side to achieve a secondary separation zone.

For the eddy viscosity of the turbulent flow, simple algebraic relations similar to those of Ref. 12 are assumed. The effective coefficient of viscosity $\mu$ and thermal conductivity $K$ are then defined as follows:

$$
\begin{gathered}
\mu=\mu_{1}+\mu_{1} \\
K=C_{p}\left(\frac{\mu_{l}}{P r_{1}}+\frac{\mu_{1}}{P r_{1}}\right)
\end{gathered}
$$

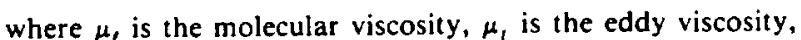
and $P r_{p}$ is the Prandtl number associated with the laminar flow (0.72). The turbulent Prandtl number Pr, is assumed to be 0.9 in the present analysis; and

$$
\begin{gathered}
\mu_{1}=\rho \frac{\rho^{2}}{r}\left[\left(\frac{\tau_{12}}{\mu}\right)^{2}+\left(\frac{\tau_{32}}{\mu}\right)^{2}\right]^{1 / 2} \\
\ell=0.09 \theta_{e} \tanh \left(\frac{0.4}{0.09} \frac{\theta}{\theta_{e}}\right) Q \\
Q=1-\exp \left(-\frac{r \theta}{A}\right), \quad A=26.0 \frac{\mu}{\rho}\left(\frac{\rho}{\tau}\right)_{w}^{1 / 2}
\end{gathered}
$$

The resultant wall shear stress is

$$
\tau_{w}=\left[\left(\tau_{12}\right)_{w}^{2}+\left(\tau_{23}\right)_{w}^{2}\right]^{1 / 2}
$$

where subscripts 12 and 23 denote $(r, \theta)$ and $(\theta, \phi)$ directions, respectively. The subscript $w$ indicates evaluation at the wall. The viscous layer edge, $\theta_{e}$, is defined to be that mesh point nearest the wall where the modulus of vorticity is less than 0.0025 times the wall value.

In the present study an approach based on previous results ${ }^{8}$ was taken. The eddy viscosity model [Eqs. (2)] was used in unmodified form in the attached region up to the vicinity of the expected primary separation line. The eddy viscosity in the separated region was reduced by a constant factor, and a linear connection bracketing the expected separation line was used to connect the reduced value in the separated region to the unmodified eddy viscosity. In equation form, this procedure is

$$
\begin{array}{rlrl}
\mu_{l_{\theta}} & =\mu_{l_{\phi}}=1.0 \mu_{1} & 0 \mathrm{deg} \leq \phi \leq \phi_{i} \\
& =\mu_{l_{\phi}}=\left[1.0-\frac{1.0-C_{2}}{\phi_{f}-\phi_{i}}\left(\phi-\phi_{i}\right)\right] \mu_{i} & \phi_{i} \leq \phi \leq \phi_{f} \\
& =\mu_{l_{\phi}}=C_{2} \mu_{i} & \phi_{f} \leq \phi \leq 180 \mathrm{deg}
\end{array}
$$

where $\phi_{\text {, }}$ and $\phi_{f}$ indicate the beginning and end of the change in the eddy viscosity distribution. The numerical value of $C_{2}$ was 0.175 . In all cases, a relaxation procedure ${ }^{13}$ was used in the cross-flow direction $\left(\mu_{1 \phi}\right)$ only:

$$
\mu_{l_{\phi}}=\mu_{\phi_{\phi_{0}}}+\left(\mu_{\phi}-\mu_{\phi_{0}}\right) \exp (-\Delta \phi / \lambda)
$$

where the relaxation parameter $\lambda=20 \theta_{e 0}$. The initial point for the relaxation was the circumferential mesh point prior to primary separation.

The incorporation of the distribution in eddy viscosity imposed by $\mathrm{Eq}$. (3) results in the computation providing a flowfield consistent wth the topological pattern presented in Fig. 1.

\section{Results}

Comparisons between flight and wind tunnel measurements are restricted herein to freestream Mach numbers of 1.5 and 1.8 at a cone angle of incidence equal to $11 \mathrm{deg}$ ( a relative incidence of 2.2 ) under nominally 0 -deg sideslip conditions. The Reynolds numbers per meter in the two test environments were typically 12 and $9 \times 10^{6}\left(4\right.$ and $\left.3 \times 10^{6} / \mathrm{ft}\right)$.

Of critical issue, when comparing flight and wind tunnel measurements, is the specification of the environments in which the measurements are made. The root-mean-square pressure fluctuation levels (as a percentage of freestream dynamic pressure) in the wind tunnel $(1.1 \%$ ) are clearly at least one order of magnitude higher than typically measured 
in the atmosphere ${ }^{9.14}(0.04 \%) \S$ over the Dryden Flight Research Center. In addition, we note that the rms mass flux levels measured on a single hot wire in the wind tunnel rise monotonically from 0.15 to $1.5 \%$ in the Mach number range 0.6-1.8. Thus, compared with the quiescent conditions of flight, the wind tunnel measurements are conducted in a freestream of nominal $1 \%$ turbulence level.

As an example of parameter variations during a typical Mach 1.5 night, Fig. 3 presents time histories of airplane Mach number, altitude, Reynolds number, angle of incidence, and angle of sideslip during a $4-\mathrm{min}$ period. The particular parameters of $M_{\infty}, \alpha$, and $\beta$ are demonstrated to vary within $\pm 0.02, \pm 0.2$, and $\pm 0.1 \mathrm{deg}$. The wind tunnel flow quality is discussed in Ref. 9.

\section{Mean Static Pressures at the Surface}

Figures 4 and 5 illustrate circumferential static pressure distributions on the cone surface at the test Mach numbers of 1.8 and 1.5. The pressures are shown for the station $76.2 \mathrm{~cm}$ (30 in.) from the cone apex, with the outputs from four orifices at 90 -deg intervals around the surface plotted in the same figure, (Fig. 4). In addition, measurements are also provided at $x=78.7,83.8$, and $88.9 \mathrm{~cm}(31,33$, and 35 in.) (Fig. 5). The pressure distributions displayed virtual symmetry about the meridian plane so that only measurements made on one side of the cone are presented.

The respective flight and wind-tunnel Mach 1.8 results at station 30 are shown in Figs. $4 a$ and $4 b$. The results are ploned as surface mean pressure coefficients vs circumferential angle, $\phi$, where the windward generator is recognized at $\phi=0 \mathrm{deg}$. The experimental results are plotted with symbols, and the computed results are plotted with a continuous line. The flight and wind tunnel data are in reasonable agreement in both trend and magnitude. The high quality of the flight data is especially noteworthy: the static pressure outputs at station 30 are virtually superimposed. However, some discrepancies appear near the windward ray and towards the leeward ray in the zone where the primary and secondary separations exist ( $\pm 140 \mathrm{deg}<\phi \leq 180 \mathrm{deg}$ ). Note the suction peak close to $\phi \pm 165 \mathrm{deg}$, which signifies the presence of the primary vortex core above the surface.

The computation duplicates the trends in the measurements, especially on the windward side. On the leeward, however, the numerical results provide a more positive level of pressure, although the suction peaks beneath the vortex cores are close to the experimental locations.

Figures $5 \mathrm{a}$ and $5 \mathrm{~b}$ portray the circumferential pressure distributions at Mach 1.5 along the cone at four axial stations, $x=76.2,78.7,83.8$, and $88.9 \mathrm{~cm}(30,31,33$, and $35 \mathrm{in}$.) from the apex. The pressures along the cone are virtually invariant, confirming the near absence of length effects at supersonic Mach numbers.

Thus, from Figs. 4 and 5 , it may be deduced that up to the flank position on the cone, the boundary layer develops from the windward ray and undergoes acceleration in a very favorable pressure gradient. Once past $\phi=100 \mathrm{deg}$, the viscous flow encounters a stiff adverse pressure gradient, departing from the surface at the primary separation line, $\phi_{s_{l}}$. The thin boundary layer that then develops from the leeward attachment line does so in the region of favorable pressure gradient from $\phi=180$ to about $165 \mathrm{deg}$. Subsequently, the flow separates at $\phi_{S_{3}}$. The experimental primary and secondary separation line positions marked in Figs. 4 and 5 are given from the pressure distributions obtained with the obstacle blocks described below. These measurements of $\phi_{s_{1}} \approx 142 \mathrm{deg}$ and $\phi_{S_{2}} \approx 157.5 \mathrm{deg}$ are demonstrated to be in close agreement at both supersonic Mach numbers; the computations, on the other hand, predict values of $\phi_{S}$, and $\phi_{S 2}$, that are marginally displaced toward the windward side.

\$At supersonic speed in a quiescent atmosphere, this is likely a probe induced perturbation level.

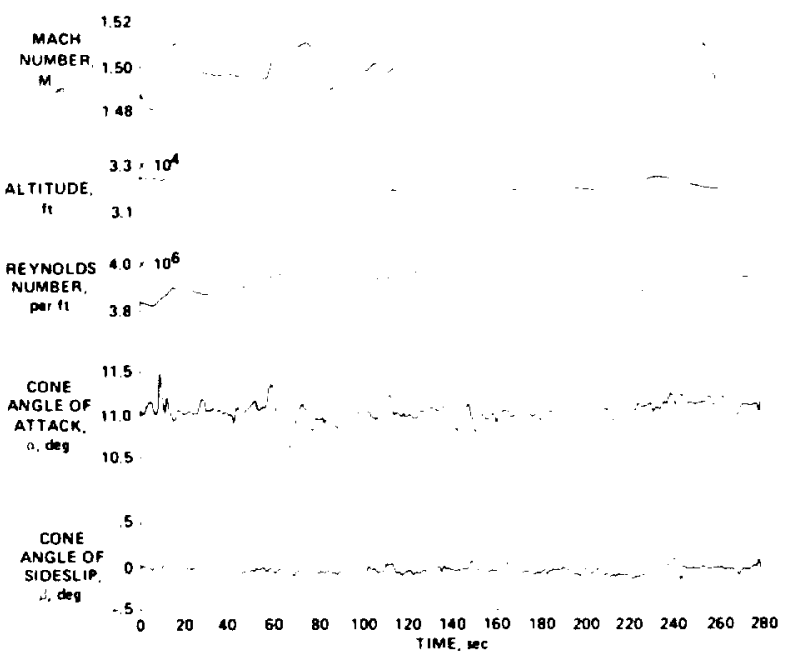

Fig. 3 Time history during a typical fight at $M_{\infty}=1.5$.

a)

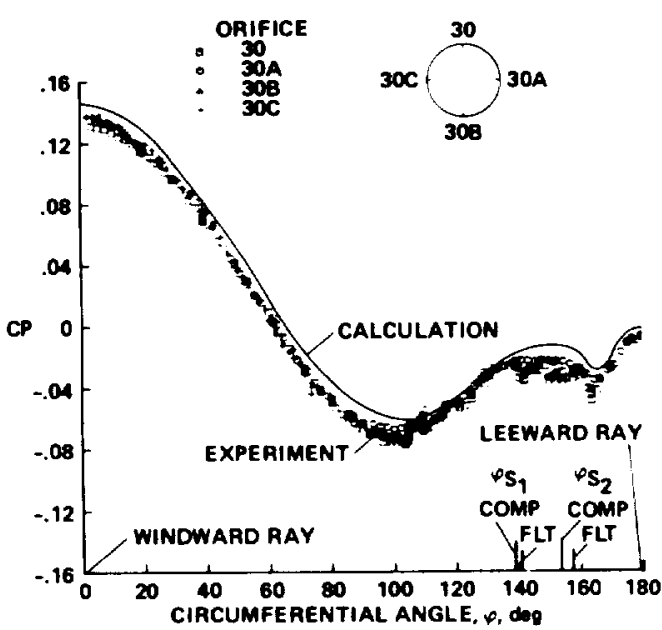

ORIFICE

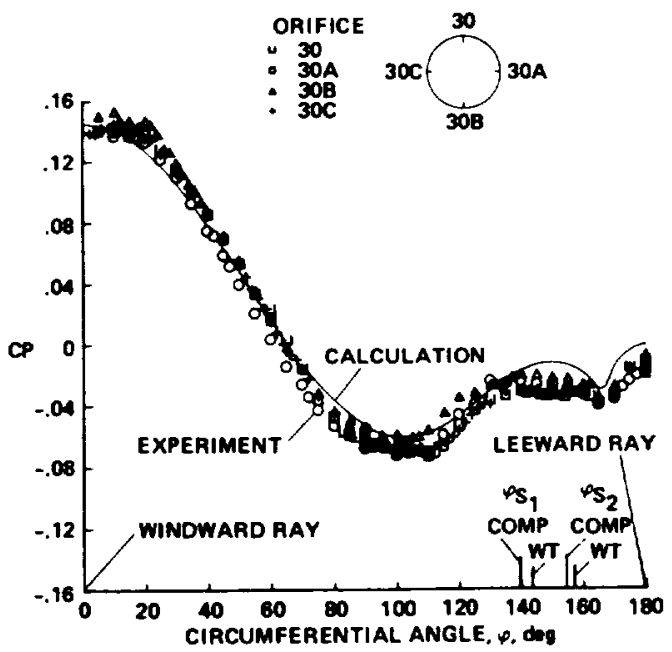

b)

Fig. 4 Flight and wind tunnel results at $M_{0}=1.8$; circumferential mean pressure distributions at $x=30$ in., $\alpha=11$ deg. a) Flight results, $R N=4.2 \times 10^{6} / \mathrm{ft}$. b) Wind tunnel results, $R N=2.6 \times 10^{6} / \mathrm{ft}$.

\section{Obstacle Block Pressures and Separation Line Position}

To determine separation line positions where use of an oil indicator on the surface is inappropriate, obstacle blocks ${ }^{15-17}$ may be used. These are small machined cuboids that are attached to the cone surface abutting static pressure orifices as shown in Fig. 6. The performance of the block is analogous to 
a)

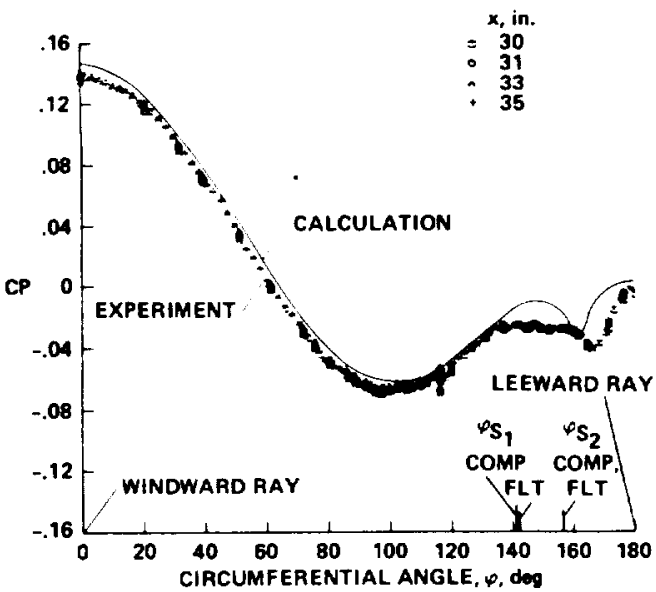

b)

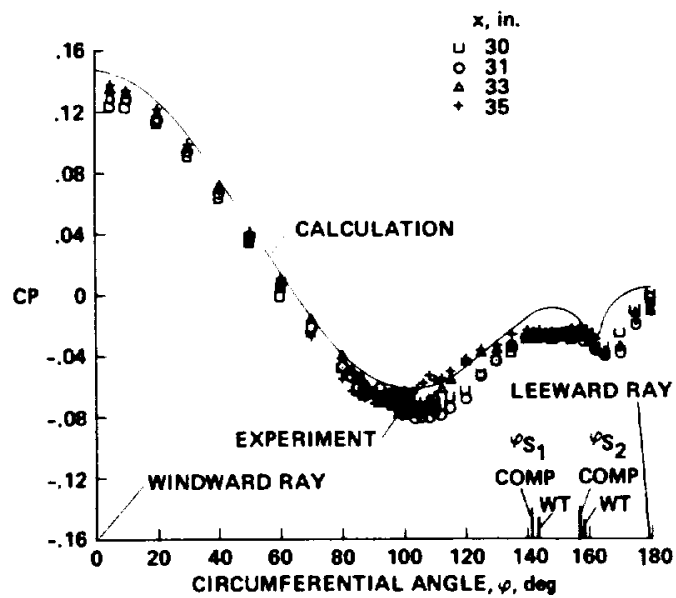

Fig. 5 Flight and wind tunnel results at $M_{\infty}=1.5$; circumferential mean pressure distributions at $x=30-35$ in., $\alpha=11$ deg. a) Flight results, $R N=4.0 \times 10^{6} / \mathrm{ft}$. b) Wind tunnel results, $R N=2.9 \times 10^{6} / \mathrm{ft}$.

that of a surface pitot tube, the signature from which is sufficiently sensitive to yield either a large increase in amplitude through a transition zone, ${ }^{14}$ or well defined troughs at three-dimensional separation lines. ${ }^{10}$

Figure 6a indicates block pressure coefficients obtained at the four pressure orifices at station 30 for the Mach 1.8 flight case. These results should be compared with the wind tunnel results for the same Mach number, which are illustrated in Fig. 6b. There is fair agreement between the flight and wind tunnel results. The pronounced troughs in the values of CPB close to $\phi=140$ and $160 \mathrm{deg}$ denote the primary and secondary separation line positions where the skin friction has a minimum but finite value. (Only at the singular points at which the separation line begins and ends is the magnitude of the local skin friction equal to zero in a three-dimensional viscous flow.) Note the elevated values of CPB toward the attachment lines where the boundary layers are thin: at the windward ray, leeward ray, and between the separation lines. The monotonic decrease in CPB level from the windward to the primary separation line location indicates the behavior of a fully turbulent viscous flow with no evidence of delayed transition effects. In the wind tunnel tests, the block outputs at stations 30 and $30 \mathrm{~B}$ were calibrated directly against Preston tubes at stations $30 \mathrm{~A}$ and $30 \mathrm{C}$ with the cone at 0 -deg incidence. ${ }^{17}$ The Preston-tube calibration for compressible flow developed in Ref. 18 (with slight modifications ${ }^{19}$ ) was used to give skin friction magnitudes. Figure $6 \mathrm{~b}$ indicates the corresponding skin-friction levels obtained from the blocks at high and low point values of CPB at $\alpha=11$ deg. Analogous results to those at Mach 1.8 were obtained at Mach 1.5 and 0.6. The measured and predicted primary and secondary separation line positions are shown in Fig. 4. a)
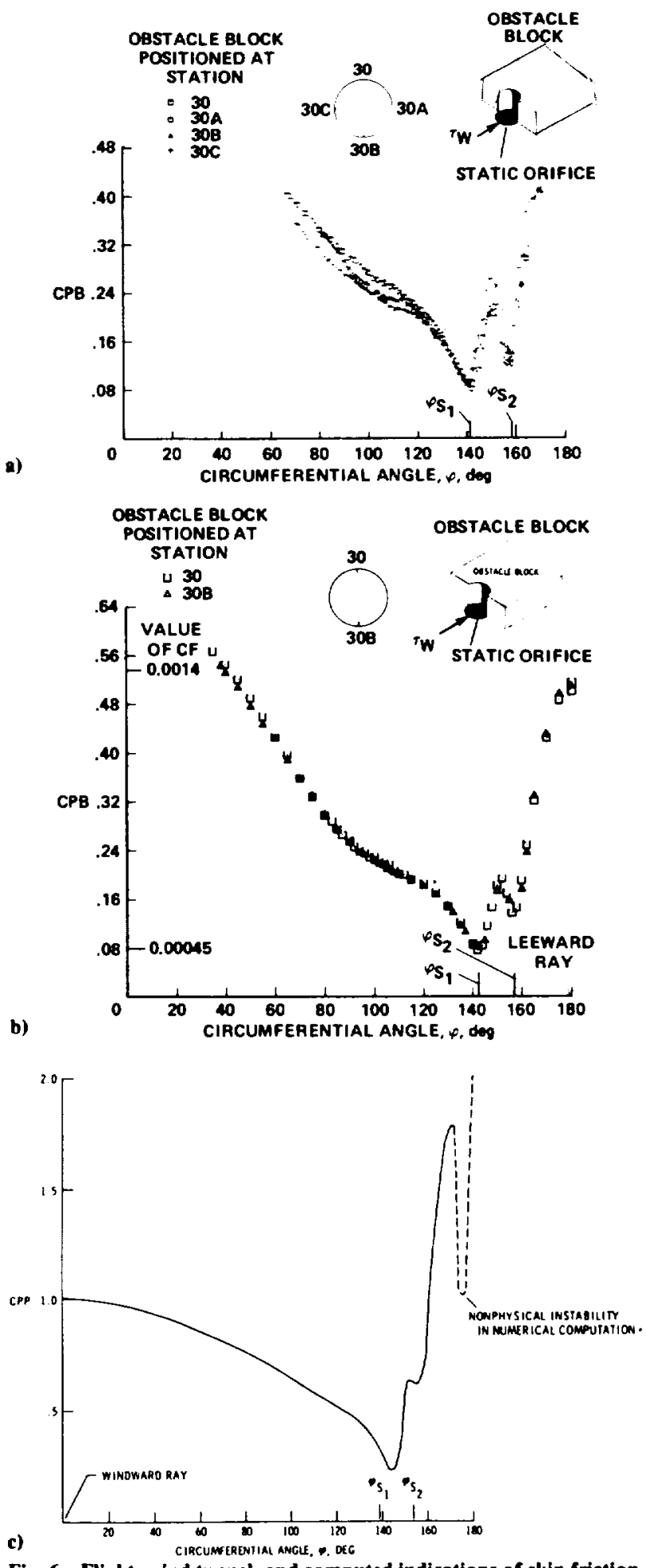

Fig. 6 Flight, wind tunnel, and computed indications of skin friction at $M_{\infty}=1.8, \alpha=11$ deg. a) Flight messurements of obstacle bjock pressures at $x=30$ in., $R N=4.2 \times 10^{6} / \mathrm{ft}$. b) Wind (unnel measurements of obstacle block pressures at $x=30$ in., $R N=2.6 \times 10^{6} / \mathrm{ft}$. c) Computation of pitot pressure at $H=0.012$ above cone surface, $R_{x \infty}=9.8 \times 10^{6}$.

The blocks clearly measure a recovery pressure at an elevation very close to the surface of the cone. For purposes of comparison with the experimental Mach 1.8 results, the computed distribution of pitot pressure coefficient, CPP, at a distance above the cone surface equal to about 0.75 the block height, is shown in Fig. 6c. Again, there are noticeable 
troughs at the circumferential locations adjacent to the computed primary and secondary separation line positions, corresponding with the positions of the dividing surfaces emanating from $\phi_{S}$ and $\phi_{S_{2}}$ (Fig. 1). Some details of the external mean flowfield are captured in the Mach $1.8 \mathrm{com}-$ puted pitot contours shown in Fig. 7. The dividing surface from the primary separation line location, $\phi_{S_{l}}$, is shown as a chain-dot line; the locus of the inflexional zero velocity point in the cross-flow velocity profiles (in the conical projection) is indicated as a dashed line. There is close qualitative agreement between this computed result and previously published measurements in the external flow. ${ }^{1,3}$ It shows the extensive domain of the primary vortex close to the leeward ray, with the vortical core immediately above the circumferential angle $\phi=166 \mathrm{deg}$, exactly where the calculated suction peak appeared in the Mach 1.8 static pressure distribution in Fig. 4a.

\section{Surface Shear Stress Directions}

The computed limiting streamline angles relative to conical rays can be compared with the experimental surface shear stress directions obtained by oil-dot surface flow visualization in the wind tunnel. To provide a complete map of the wall shear stress directions, precisely cut thin Mylar film was affixed to the cone surface, and oil dots were applied around the circumference at given axial stations prior to a tunnel run. After the run, the Mylar was carefully unwrapped from the cone and photographed to give a flat projection. Figure 8 presents a section of one of these photographs taken for a Mach 1.8 run at 12.5 -deg incidence in the wind tunnel. This angle is higher than the 11 -deg test condition presented thus far; the skin friction line map, however, is qualitatively identical at both angles of incidence. The limiting flow at the base of the three-dimensional boundary layer sweeps around the cone from the windward ray, and then turns to approach asymptotically the primary separation line in the region of circumferential adverse pressure gradient (Fig. 4b). The leeward boundary layer grows outboard from the leeward meridian (a line from which the adjacent skin friction lines diverge) to approach asymptotically the secondary separation line. As Fig. 8 shows distinctly, there is another reattachment line between the primary and secondary separation lines (see also Fig. 1).

The computed limiting streamline angles are drawn in Fig. 8 for a Navier-Stokes calculation equivalent to the experimental conditions. The computed separation lines are shown as solid lines and reattachment lines by dashed lines. The lines are repeated at the base of the cone for clarity. The agreement between the computational results and the experiment is good.

\section{Fluctuating Pressure Measurements on the Cone Surface}

Microphone and Kulite pressure signatures were obtained at $\alpha=11 \mathrm{deg}$ in flight and in the wind tunnel at the station
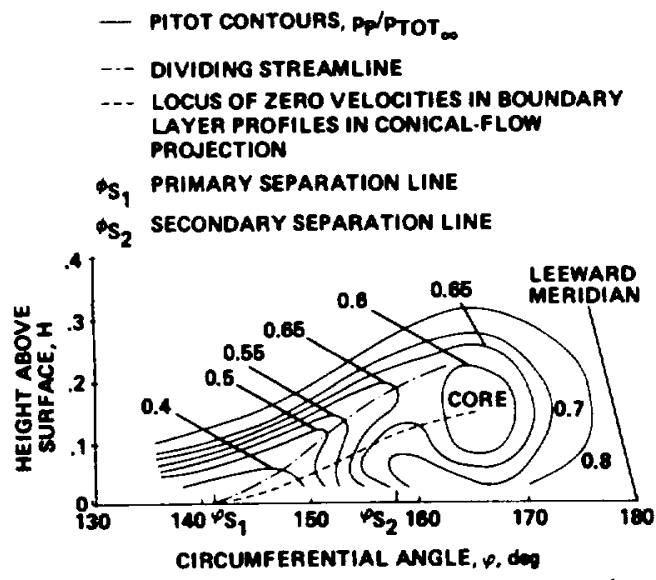

Fig. 7 Computed contours of consiant pitot pressure on leeward of cone at $M_{\infty}=1.8, \alpha=11 \mathrm{deg}, R_{x \infty}=9.8 \times 10^{6}$.
$86.4 \mathrm{~cm}$ (34 in.) from the apex as the cone was rolled about its axis. All microphone and Kulite data provided the same qualitative trends in a given test but absolute levels did vary slightly, perhaps as a result of minute differences in flushness of the installation of the sensors in the cone surface. Dynamic calibration of the sensors (in the absence of freestream flow) produced repeatable and consistent signal responses before and after the tests.

Measurements from one microphone obtained at Mach 1.5 in the wind tunnel are shown in Fig. 9 as rms values of the surface pressure fluctuation, $\langle p\rangle$, normalized by the freestream mean dynamic pressure, $q_{\infty}$. The characteristic feature of these measurements is the progressive reduction in signal amplitude as the primary separation line, $\phi_{S_{I}}$, is approached, with a similarly low level at the secondary separation line, $\phi_{S_{2}}$. This feature was found at all Mach numbers and was witnessed in an earlier investigation. "The signal amplitude climbs again towards the leeward attachment line to a level close to that on the windward ray attachment line. Not understood, however, is the reason for the changing amplitudes in the attached flow around the flank of the cone.

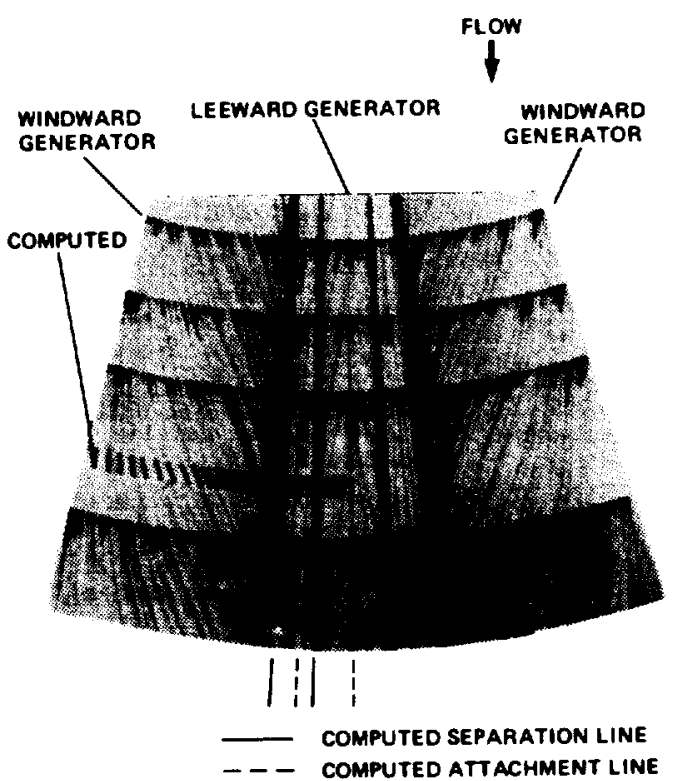

Fig. 8 Unwrapped skin-friction line pattern obtained in wind tunnel compared with computed limiting streamline directions at $M_{\infty}=1.8$, $\alpha=12.5$ deg.

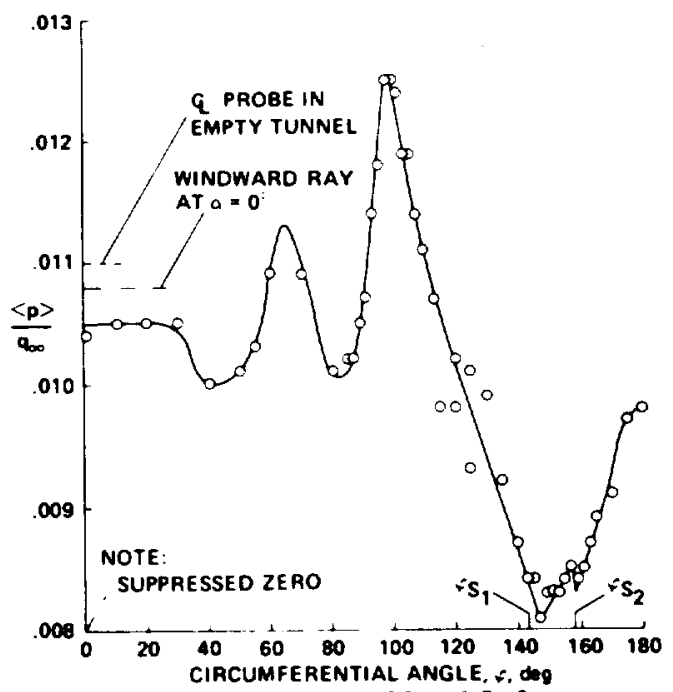

Fig. 9 Wind tunnel measurements at $M_{\infty}=1.5$ of root-mean-square microphone output at $x=34 \mathrm{in} ., \alpha=11 \mathrm{deg}, R N=2.9 \times 10^{6} / \mathrm{ft}$. 


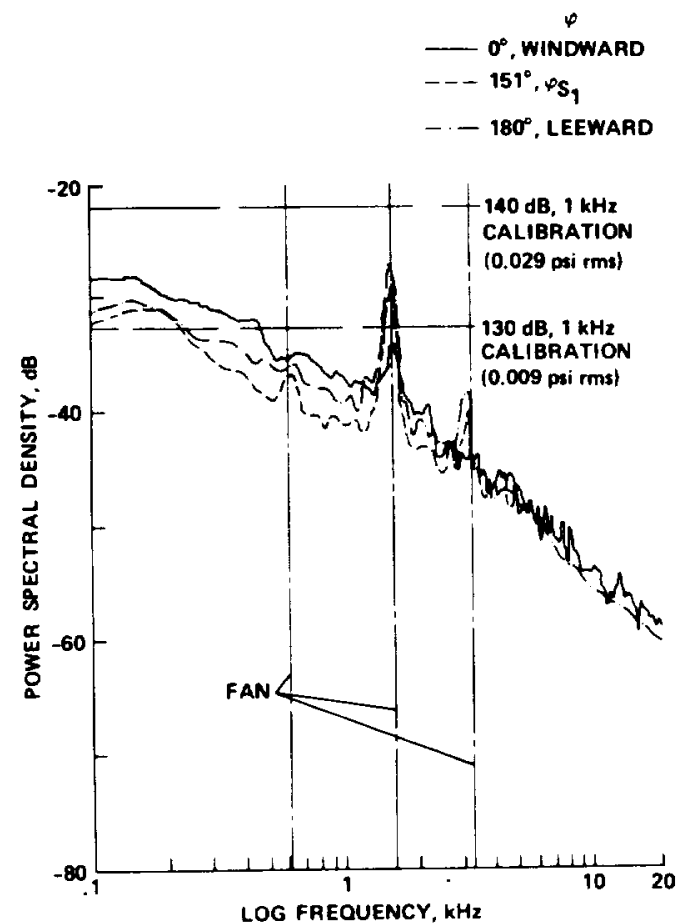

Fig. 10 Wind tunnel pawer spectral density measurements at windward and leeward rays compared with primary separation line, $M_{\infty}=1.5, R N=2.9 \times 10^{6} / \mathrm{ft}$.

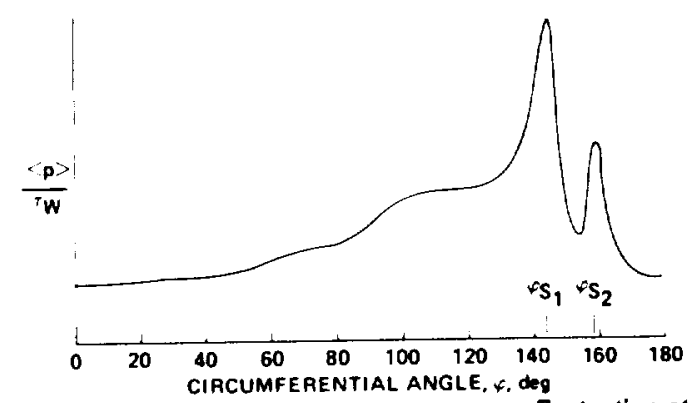

Fig. 11 Wind tunnel root-mean-square pressure nuctuation at cone surface normalized by local wall shear stress, $M_{\infty}=1.5, \alpha=11 \mathrm{deg}$, $R N=2.9 \times 10^{6} / \mathrm{ft}$.

Note that the fluctuation level on the windward ray at $\alpha=11$ deg, is close to that measured at $\alpha=0 \mathrm{deg}$, and to the freestream fluctuating static pressure in the empty tunnel. The latter was measured with a Kulite pressure transducer situated within a small tangent-ogive/cylinder probe body. The flight measurements yielded signal levels typically one order less than in the wind tunnel, with much less relative distinction between those levels at the separation and attachment lines.

Figure 10 shows sample wind tunnel power-spectral density results indicating a reduced energy level at primary separation relative to the windward and attachment line boundary layers. When, on the other hand, the root-mean-square pressure output is normalized by the local value of skin friction, characteristic peaks" are demonstrated at the primary and secondary separation lines. In other words, as separation is approached, the local skin friction decreases at a faster rate than does the root-mean-square pressure fluctuation. We see that the spiky region in Fig. 9 does not translate into a similarly obvious region in Fig. 11; rather, there is a relatively smooth enhancement of $\left(\langle p\rangle / \tau_{w}\right)$ in the $\phi$ range $40 \mathrm{deg}$ $<\phi<120 \mathrm{deg}$. Note that there is a noticeable peak in the wind tunnel spectral outputs in Fig. 10 at $1.6 \mathrm{kHz}$. This appears to be a harmonic of the fan rotational speed, rather than a discrete tone from the slots in the tunnel working section since

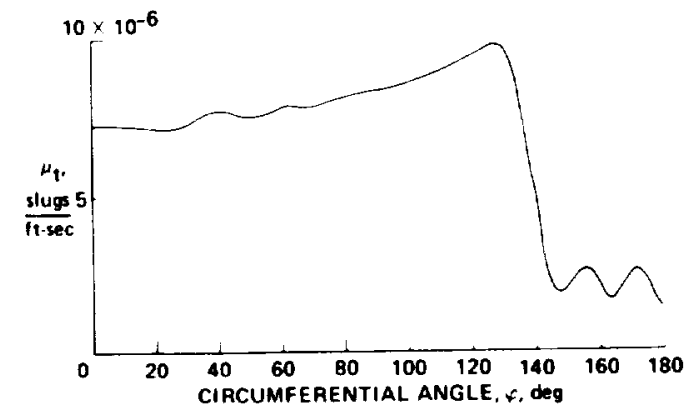

Fig. 12 Circumferential variation of eddy viscosity in numerical computation, $M_{\infty}=1.5, \alpha=11$ deg, $R_{x_{\infty}}=9.8 \times 10^{6} / f 1$.

the same peak was also observed on the $\alpha=0$-deg runs, with and without the slots sealed.

\section{Eddy Viscosity Turbulence Model}

In an earlier numerical computation, ${ }^{10}$ the eddy viscosity model was tailored to suit cone results obtained in the National Aeronautical Establishment (NAE) $5 \times 5 \mathrm{ft}$ $(1.5 \times 1.5-\mathrm{m})$ Blowdown Wind Tunnel at Ottawa, ${ }^{1}$ where the coefficient $C_{2}$ was set at a value of 0.3 . Attempting to modify the eddy viscosity so that the circumferential static pressures tended toward the flight data required $C_{2}$ to be reduced to 0.175 . In so doing, a nonphysical instability developed in the computation of the flowfield near the leeward ray (Fig. 6c). The instability was believed to result from the unfavorable interaction of the small value of eddy viscosity (see Fig. 12) with the scheme of the numerical solution. The implication is that the eddy viscosity should be increased again as the leeward attachment line is approached, because in this region the properties of the leeward boundary layer are similar to those of the windward attachment line flow. The eddy viscosity would then follow the trend of the root-mean-square pressure signature as depicted in the $\phi$ range $120 \mathrm{deg}<\phi<180$ deg shown in Fig. 9. A recent computation indicates that such a change does indeed reduce the instability.

\section{Conclusions}

A 5-deg semiangle cone was tested at 11-deg angle of incidence and at Mach numbers of $1.8,1.5$, and 0.6 in flight and in the Ames $6 \times 6-\mathrm{ft}(1.8 \times 1.8-\mathrm{m})$ Wind Tunnel under turbulent viscous flow conditions. The experiments had the following results.

1) The measured static pressures on the cone surface in the two test environments agreed reasonably well. Computed results at Mach 1.8 and 1.5 where conical now approximations to the Navier-Stokes equations were invoked, and where a tailored eddy-viscosity distribution around the circumference was used, provided the correct trends in the pressure distributions, but had generally slightly more positive values.

2) The attachment of obstacle blocks abutting the cone surface static pressure orifices to measure an effective surface pitot pressure facilitated the detection of primary and secondary separation lines at well-defined troughs in the block pressure distributions. The results from flight and from the wind tunnel were again in agreement. The numerical computations in supersonic flow also provided separation line positions close to the measured ones.

3) The rms fluctuating pressure distribution on the cone surface in the wind tunnel supported previous modifications in trend made to the turbulent eddy viscosity model in numerical computations; namely, a sharp fall in amplitude of each quantity upon passage through the primary separation zone. To improve the model, it is proposed that the eddy viscosity should again be increased as the leeward ray is approached, which is consistent with the wind tunnel measurements. Notwithstanding, the wind-tunnel root-mean- 
square pressure signatures were typically one order larger than the magnitudes registered in flight.

\section{References}

'Rainbird, W. J., "The External Flow Field About Yawed Circular Cones," Hypersonic Boundary Layers and Flow Fields, AGARD-CP-30, May 1968, pp. 19-1-19-10.

${ }^{2}$ Nebbeling, C. and Bannink, W. J., "Experimental Investigation of the Supersonic Flow Field About a Slender Cone at High Incidences," Rept. LR-233, Delft University of Technology, Dept. of Aerospace Engineering, the Netherlands, Nov. 1976.

${ }^{3}$ Peake, D. J., Owen, F. K., and Johnson, D. A., "Control of Forebody Vortex Orientation to Alleviate Side Forces," AlAA Paper 80-0183, Jan. 1980.

"Crabbe, R. S., "Flow Separation About Elliptic Cones at Incidence," Aero. Rept. LR-436, National Research Council of Canada, Aug. 1965.

${ }^{5}$ Peake, D. J. and Tobak, M., "Three-Dimensional Interactions and Vortical Flows with Emphasis on High Speeds," AGARD-AG252, July 1980; also NASA TM-81 169, 1980.

"Lighthill, M. 'J., "Introduction: Boundary Layer Theory," Laminar Boundary Layers, edited by L. Rosenhead, Oxford University Press, England, 1963, pp. 46-113.

${ }^{7}$ Legendre, R., "Ecoulement Au Voisinage de la Pointe Avant d'une Aile à Forte Fleche aux Incidences Moyennes," La Recherche Aéronautique, No. 30, 1952, pp. 3-8; No. 31, 1953, pp. 3-6; No. 35, 1953 , pp. 7.8.

I McRae, D. S. and Hussaini, M. Y., "Numerical Simulation of Supersonic Cone Flow at High Angle of Attack," High Angle of Altack Aerodynamics, AGARD-CP-247, Oct. 1978, pp. 23-1-23-10.

${ }^{9}$ Peake, D. J., Fisher, D. F., and McRae, D. S., "Flight Experiments with a Slender Cone at Angle of Attack," AlAA Paper 81 0337, Jan. 1981.
${ }^{10}$ McRae, D. S., Peake, D. J., Fisher, D. F., "A Computational and Experimental Study of High Reynolds Number Viscous/Inviscid Interaction About a Cone at High Angle of Attack," AIAA Paper 801422, July 1980 .

"Peake, D. J., Owen, F. K., and Higuchi, H., "Symmetrical and Asymmetrical Separations About a Yawed Cone," High Angle of Altack Aerodynamics, AGARD-CP-247, Oct. 1978, pp. 16-1-16-27.

${ }^{12}$ Harris, J. E. and Morris, D. L., "Solution of the ThreeDimensional Compressible, Laminar and Turbulent Boundary-Layer Equations with Comparisons to Experimental Data," Proceedings of the Fourth International Conference on Numerical Methods in Fluid Dynamics, June 1974, pp. 204-211.

${ }^{13}$ Shang, J. S., Hankey, W. L. Jr., and Law, C. H., "Numerical Simulation of Shock Wave-Turbulent Boundary-Layer Interaction," AIAA Journal, Vol. 14, Oct. 1976, pp. 1451-1457.

${ }^{14}$ Dougherty, N. S. Jr. and Fisher, D. F., "Boundary-Layer Transition on a 10 Deg Cone: Wind Tunnel/Flight Correlation," AIAA Paper 80-0154, Jan. 1980.

${ }^{15}$ Nituch, M. J., "The Use of Congruent Obstacle Blocks for the Indirect Measurement of Turbulent Skin Friction on Smooth Surfaces," Masters Thesis, Carleton University, Ottawa, Canada, Aug. 1972.

${ }^{16}$ Peake, D. J., "Three-Dimensional Swept Shock/Turbulent Boundary-Layer Separations with Control by Air Injection," Aero. Rept. LR-592, National Research Council of Canada, July 1976.

${ }^{17}$ Elfstron, G. M., Peake, D. J., et al., "The Obstacle Block as a Device to Measure Turbulent Skin Friction in Compressible Flow," AIAA Paper 82-0589, May 1982.

${ }^{18}$ Bradshaw, P. and Unsworth, K., "A Note on Preston Tube Calibrations in Compressible Flow," IC-Aero-73-07, Imprial College of Science and Technology, Sept. 1973.

${ }^{19}$ Bradshaw, P., private communication from Imperial College of Science and Technology, Oct. 1978. 